\title{
5. Methodisches Vorgehen
}

Mein Erkenntnisinteresse lautet: Inwieweit ist barrierefreier bzw. -reduzierter Wohnraum ein Handlungsfeld für die Politik? Ein zweigleisiges Forschungsdesign nähert sich diesem Erkenntnisinteresse und orientiert sich an den folgenden beiden Forschungsfragen:

Frage A: In welchen Ausprägungen nutzen die Bundesländer politische Maßnahmen (»Policies«) zur Steuerung von barrierefreiem bzw. -reduziertem Wohnraum? Frage B: Was erklärt das Zustandekommen bzw. Ausbleiben sowie die konkrete Ausgestaltung dieser Policies? Zur Beantwortung der Frage A wird mithilfe einer Typologie der bestehende Policy-Mix abgebildet und damit der Untersuchungsgegenstand operationalisiert (Kapitel 6). Für die Frage B arbeiten die Fallstudien zu Sachsen-Anhalt und Hessen die politischen Prozesse heraus, welche hinter diesem Policy-Mix liegen (Kapitel 7). Da dieses zweigleisige Vorgehen zwei unterschiedlichen methodischen Ansätzen folgt, sind diese je einzeln zu erläutern (Kapitel 5.1 zur Typologie und Kapitel $5.2 \mathrm{zu}$ den Fallstudien). Vorab wird aber noch in das grundlegende Forschungsdesign eingeführt.

Abbildung 10: Erkenntnisinteresse via zweigleisigem Forschungsdesign

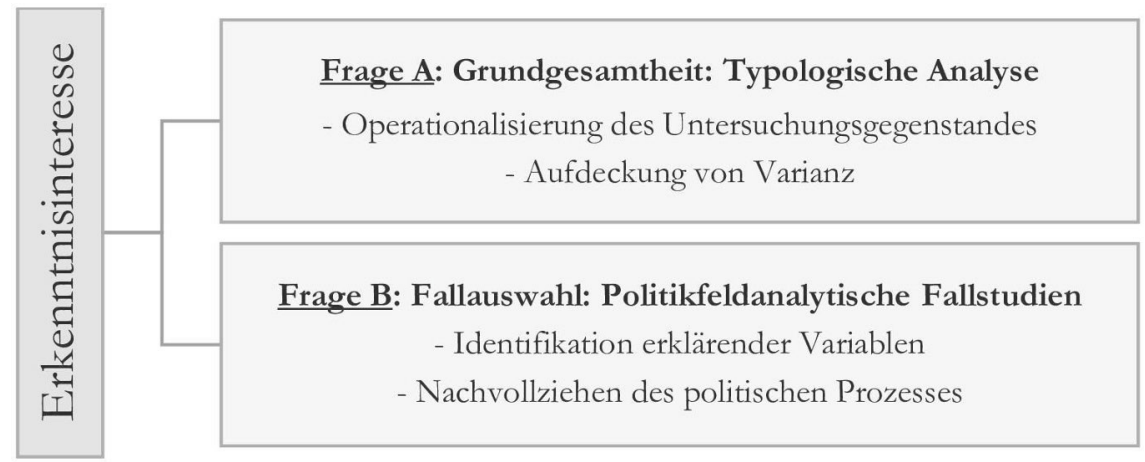


Abbildung 10 stellt die beiden die Forschungsarbeit leitenden Forschungsfragen dar. Die typologische Analyse zur Frage A untersucht die Grundgesamtheit aller 16 Bundesländer, die Politikfeldanalyse in Frage B prüft tiefergehende kausale Zusammenhänge. ${ }^{1}$ Idealtypisch beginnt der Forschungsprozess bei der Grundgesamtheit und den darin umgesetzten Policies, liegt doch das Ziel dieses Forschungsschrittes in einer Strukturierung des Untersuchungsgegenstandes selbst. Darauf aufbauend analysieren die Fallstudien die in einzelnen Bundesländern ablaufenden politischen Prozesse. Diese Trennung ist indessen eine analytische: Der tatsächliche Forschungsprozess wechselt zwischen beiden Forschungsfragen. Dies soll im Folgenden mit Rückgriff auf Grundannahmen aus der qualitativen Sozialforschung verdeutlicht werden.

Die vorliegende Forschungsarbeit verortet sich in der qualitativ-empirischen Politikanalyse, wobei nach Egner (2019a: 36) das Verstehen eine "wichtige Vorstufe zum Erklären« sein kann. Die Entwicklung einer Typologie zu politischen Maßnahmen zum barrierefreien bzw. -reduzierten Wohnraum leistet den ersten Schritt des Verstehens. Darauf aufbauend kann sich im Rahmen der Fallstudien der Erklärung gewidmet werden. Dieses scheinbare Nacheinander von Verstehen und Erklären soll jedoch nicht über den verfolgten zirkulären Forschungsprozess hinwegtäuschen: »Phasen der Datenauswahl, Datenerhebung und Datenanalyse wechseln sich iterativ mit Phasen der theoretischen Reflexion ab« (Baur/Blasius 2019: 8). So besteht ein ständiges Wechselverhältnis zwischen der Operationalisierung des Untersuchungsgegenstandes und den Fallstudien, da erst das Datenmaterial aus den Fallstudien zum vollständigen Verständnis des Untersuchungsgegenstandes selbst verhilft (vgl. Kapitel 5.2.2). Das Datenmaterial wird in einer Triangulation aus Quellen- und Dokumentenanalysen, bestehenden Sekundärdaten und leitfadengestützten Interviews mit Expert*innen sowie an den politischen Prozessen beteiligten Akteuren generiert. Diese Kombination verschiedener Datenquellen soll dazu verhelfen, verschiedene Zugänge auf denselben Untersuchungsgegenstand zu beleuchten und »der Vielschichtigkeit des Untersuchten durch die Erweiterung der [...] Perspektiven umfassender gerecht zu werden « (Flick 2019: 480, vgl. auch Wolf 2015: 483; Jahn 2013: 216; Pickel 2009: 518ff.). Theorie und Empirie befinden sich dabei in einem fortwährend $\mathrm{zu}$ überprüfenden Wechselspiel: Welches sind tatsächlich diejenigen theoretischen Annahmen, die das vorliegende Erkenntnisinteresse möglichst gut erfassen (Egner 2019a: 32f., 56f.; Blatter et al. 2007: 138)? In diesem Vorgehen gelten die Gütekriterien der qualitativen Sozialforschung als handlungsleitend (vgl. Kapitel 5.3) und werden im Anschluss an die empirische Arbeit einer kritischen Reflexion unterzogen (vgl. Kapitel 8).

\subsection{Typologie politischer Instrumente}

Zur Operationalisierung und Analyse des Untersuchungsgegenstandes »Policies des barrierefreien bzw. -reduzierten Wohnraums« wird eine Typologie entwickelt und mit 
Inhalt gefüllt (vgl. Kapitel 6). Dafür ist zunächst die Auswahl der in die Typologie einfließenden Maßnahmen zu begründen (Kapitel 5.1.1), um daraufhin die Datenbasis zu erläutern, mithilfe derer die Typologie für die 16 Bundesländer in ihren konkreten PolicyAusprägungen ausgearbeitet wird (Kapitel 5.1.2).

\subsubsection{Auswahl der Policies}

Kapitel 4.2.1 hat die Typologie politischer Instrumente nach Böcher/Töller (2012: 74ff.) erörtert. Diese wird nun mit konkreten politischen Maßnahmen zum barrierefreien bzw. -reduzierten Wohnraum gefüllt, sodass am Ende ein Policy-Mix als Untersuchungsgegenstand vorliegt. Die Auswahl der Policies ist in Abbildung 11 dargestellt: Ein Dreiklang aus dem Bauordnungsrecht (regulativ), Förderprogrammen der Landesförderbanken (ökonomisch) sowie Wohnberatungsstellen zur Wohnungsanpassung (informationell).

Während die Landesbauordnungen verbindlich festschreiben, welche Standards zur Barrierefreiheit bei Bauvorhaben einzuhalten sind, schaffen die Landesförderprogramme einen finanziellen Anreiz für Umbaumaßnahmen im Bestand oder für Neubauvorhaben. Die Wohnberatungsstellen adressieren zuvorderst den Bestandsumbau, indem sie über technische und finanzielle Realisierungsmöglichkeiten informieren. Damit sind drei Maßnahmen gewählt, die das Spektrum an politischer Steuerung vom unmittelbar bindenden Ordnungsrecht bis zum weichen informationellen Instrumentarium abdecken. Wichtig bei der Auswahl war es, dass die jeweiligen Policies in möglichst allen 16 Bundesländern eingeführt wurden - nur eben in unterschiedlichen Ausprägungen.

Abbildung 11: Auswahl der Policies für die Typologie

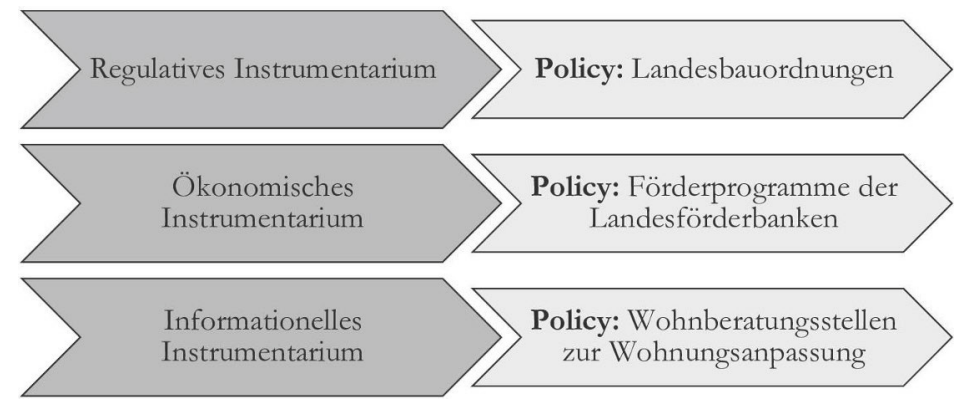

Inwieweit kooperative Maßnahmen und "Marktlösungen« als wirtschaftliche Selbstregelung beim barrierefreien bzw. -reduzierten Wohnraum vorliegen (vgl. Kapitel 4.2.1), ist im Rahmen der Fallstudien zu prüfen. Eine vollwertige Aufarbeitung für alle 16 Bundesländer wäre nicht mit den Ressourcen des vorliegenden Dissertationsvorhabens zu leisten gewesen. 


\subsubsection{Datenbasis und -auswertung}

Mit der Auswahl der Policies ist auch die Prüfung der vorhandenen Datenlage verbunden, mithilfe derer die Maßnahmen in ihren Ausprägungen angemessen untersucht werden können. Zunächst steht das Forschungsvorhaben dabei aber vor der Herausforderung, sich mit einem sogenannten moving target zu beschäftigen. Politikfelder entwickeln sich weiter, womit die Festlegung eines Untersuchungszeitraums notwendigerweise exklusiv ist und den Blick auf weitere, außerhalb dieses Untersuchungszeitraums liegende Entwicklungen verschließt. Diesem Umstand zum Trotz ist bei vergleichend angelegten Forschungsdesigns ein analoger zeitlicher Rahmen unabdingbar: Für das Jahr 2019 sind die zu erklärenden Policies aller 16 Bundesländer zu prüfen. Dieses Jahr bildet den Status Quo des Untersuchungsgegenstandes, dabei gilt für die Landesbauordnungen (Kapitel 6.1) und Förderprogramme (Kapitel 6.2) der Monat Juni 2019 als Stichdatum, für die Wohnberatungsstellen der Oktober 2019 (Kapitel 6.3).

Abbildung 12: Auswahl der Policies für die Typologie inkl. Datenbasis

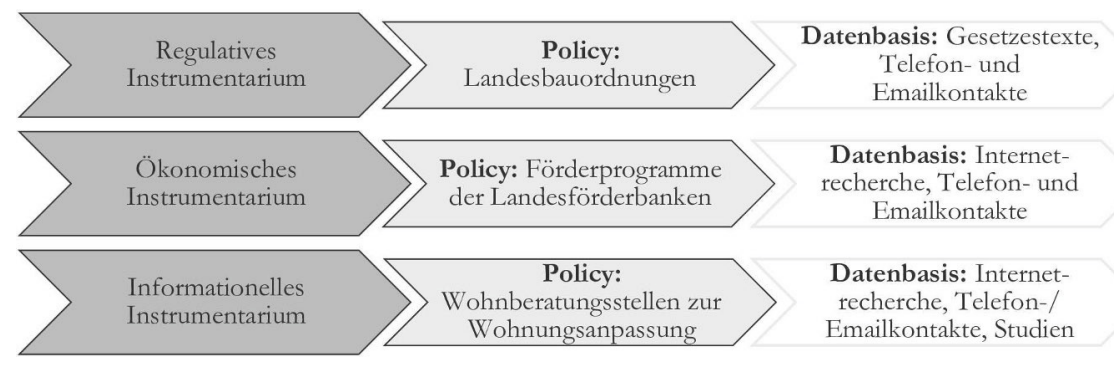

Die genutzte Datenbasis variiert je nach Policy (vgl. Abbildung 12): Während für die Landesbauordnungen die Gesetzestexte prinzipiell leicht zugänglich sind, gestaltet sich die Datenbeschaffung bei den Förderprogrammen und Wohnberatungsstellen schwieriger. Hier erfolgt zunächst eine Recherche über die Webseiten der Landesförderbanken sowie - sofern vorhanden - der öffentlichen oder privaten Akteure der Wohnberatung. Daraufhin werden die entsprechenden Akteure kontaktiert, um die gewonnenen Erkenntnisse zu validieren und bei Bedarf zu ergänzen. Im Folgenden werden die Schritte der Datenrecherche bzw. -erhebung je für die drei Policies dargelegt.

Datenbasis und Strukturierung bei den Landesbauordnungen

Für das Bauordnungsrecht stellt die Musterbauordnung einen geeigneten Startpunkt dar. Dieses Muster wird im Rahmen der Bauministerkonferenz der 16 Länder erarbeitet und bildet damit eine Art gemeinsamen Nenner (vgl. Kapitel 6.1.2). Die Landesbauordnungen selbst sind direkt über die Gesetzestexte einsehbar und können für ihre Einschätzung durch parlamentarische Dokumente zu öffentlichen Anhörungen, Plenar- und Ausschusssitzungen ergänzt werden. Dieses Material liefert wertvolles Hintergrundwissen und weist bereits auf politische Kontroversen hin, was wiederum eine Vorarbeit für die Fallstudien darstellt. Die Vorgaben der Landesbauordnungen 
sind noch in dahinterliegenden Technischen Baubestimmungen konkretisiert, weshalb auch ein Blick in diese Verwaltungsvorschriften unerlässlich ist.

Zur Validierung der Erkenntnisse und zur Klärung technischer Fragen verhelfen drei Expert"innen-Interviews. Im Sinne eines zirkulären Forschungsprozesses werden diese Expert*innen aus den Interviewpartner*innen der Fallstudien ausgewählt (vgl. Kapitel 5.2.2) und sind als Sachverständige*r für Barrierefreies Bauen fortgebildet oder im Rahmen ihrer Architektentätigkeit auf diesen Bereich spezialisiert. Um die landesspezifischen Feinheiten angemessen aufzuarbeiten, wird je ein Interview für Hessen und Sachsen-Anhalt geführt sowie eines übergeordnet mit einer Expertin aus einem dritten Bundesland. Ferner dienen Informationen aus den Fallstudien selbst zum tieferen Verständnis der bauordnungsrechtlichen Regelungen (vgl. Faktencodes in Kapitel 5.2.3).

Die Auswertung orientiert sich in ihrer Struktur an den einschlägigen Paragraphen der Bauordnungen zum barrierefreien Bauen (vgl. Kapitel 6.1.2 und 6.1.3). Dabei kommt denjenigen Inhalten eine besondere Aufmerksamkeit zu, die im politischen Diskurs Kontroversen ausgesetzt sind und die Varianzen zwischen den 16 Bauordnungen aufweisen.

Datenbasis und Strukturierung bei den Förderprogrammen

Die primäre Datenbasis für die Analyse der Förderprogramme bilden die ministeriellen Förderrichtlinien sowie die Produkt- und Merkblätter der Landesförderinstitute. Auch hier liegt mit dem KfW-Programm »Altersgerecht Umbauen« eine Referenz auf Bundesebene vor (vgl. Kapitel 6.2.2) und zur Strukturierung der Analyse werden handlungsleitende Kriterien ausgearbeitet, entlang derer sich die Analyse strukturiert (vgl. auch BMVBS 2011: 70ff.; KDA/Wüstenrot Stiftung 2014a; Schneider 2013). Vereinfacht gesagt geht es um eine Beantwortung dreier Fragen: Wer wird gefördert, was wird gefördert, wie wird gefördert? Darüber hinaus interessiert, inwieweit Barrierefreiheit bzw. -reduzierung Bestandteil größerer Programme ist oder ob explizite Zielgruppen-Programme bestehen.

Derartige Kriterien sollten sich hypothetisch aus der Dokumentenanalyse erschließen lassen, jedoch bedürfen insbesondere die Förderkonditionen zusätzlicher Nachfrage. Entsprechend validieren, falsifizieren oder ergänzen vertiefende telefonische bzw. schriftliche Kontakte mit den Förderinstituten die Rechercheergebnisse. Hierfür werden die einzelnen Förderbanken per Email kontaktiert, sofern möglich bereits konkret an Ansprechpartner*innen aus den Abteilungen für Wohnraumförderung gerichtet. Da die WIBank in Hessen und die Investitionsbank in Sachsen-Anhalt ohnehin im Rahmen der Fallstudien interviewt werden, gehen letztlich Kontaktanfragen an die 14 verbleibenden Förderbanken heraus. Mit 13 dieser 14 Institute kommen je ein bis zwei Kontakte zustande. Die darin geklärten Detailfragen beziehen sich je auf die einzelnen Förderprogramme und sind damit zu spezifisch, dass sie hier komprimiert aufbereitet werden könnten.

Letztlich beruht die Analyse zur sozialen Wohnraumförderung damit auf einer Kombination aus einer Dokumentenanalyse (Merkblätter/Produktblätter, Richtlinien) und Gesprächsprotokollen der geführten Telefonate bzw. Inhalten aus der Emailkom- 
munikation (je als Quelle »LFI« für Landesförderinstitut). Zudem fließen Erkenntnisse aus den Fallstudien-Interviews mit der hessischen WIBank und der sachsen-anhaltischen Investitionsbank sowie mit der KfW-Bank und dem Bundesinnenministerium in die Ausarbeitung ein (vgl. Kapitel 6.2.2). Die in Kapitel 6.2.3 aufbereiteten Inhalte der Landesförderprogramme zum barrierefreien bzw. -reduzierten Wohnraum basieren auf der Auswertung einer umfangreichen Matrix, die auf Nachfrage bei der Autorin einsehbar ist.

Datenbasis und Strukturierung bei den Wohnberatungsstellen

Die empirische Untersuchung der politischen Bedeutung der Wohnberatungsstellen zur Wohnungsanpassung stellt eine methodische Herausforderung dar, da ihre politische Steuerung weder in Gesetzestexten noch in Richtlinien strukturiert nachzuvollziehen sind. Teils handelt es sich auch um eine wirtschaftliche Selbstregelung, da die Beratungen vollends über Träger der freien Wohlfahrtspflege, Architektenkammern oder Wohnungsunternehmen übernommen werden. Viele Bundesländer fördern derartige Beratungsstrukturen aber in finanzieller oder organisatorischer Form, sodass durchaus von einer politischen Steuerung zu sprechen ist. Um sich dieser undurchsichtigen Struktur zu nähern, kann auf einige Studien zu Wohnberatungsstellen zurückgegriffen werden (BMVBS 2011; BMVBS 2013; Schneider 2013; KDA/Wüstenrot Stiftung 2014a; Lehrmacher-Dubberke et al. 2015; Joo 2018).

Somit basiert diese Analyse zunächst auf der vorhandenen Studienlage, welche aufbereitet und über Expert*inneninterviews mit Wohnberater*innen validiert, aktualisiert und auf den eigenen Schwerpunkt der politischen Prozesse hin konkretisiert wird. Über die Bundesarbeitsgemeinschaft Wohnungsanpassung e.V. lassen sich regionale Ansprechpartner*innen mit einem Überblick über die Strukturen im eigenen Bundesland identifizieren. Durch schriftliche Anfragen kommen telefonische, persönliche und teils vertiefende schriftliche Kontakte mit insgesamt sechs Bundesländern zustande (die Gespräche finden zwischen Oktober 2019 und Februar 2020 statt). Ferner sind auch hier Informationen aus den beiden Fallstudien zu nutzen, in dessen Rahmen ohnehin mit sachsen-anhaltischen und hessischen Wohnberater*innen gesprochen wird (vgl. Kapitel 5.2.2). Die Auswertung erfolgt entlang des erstellten Leitfadens in der Kapitelstruktur der Kapitel 6.3.1 bis 6.3.3.

Zusammengefasst orientiert sich die Datenbasis aus vorhandenen Sekundärdaten und erhobenen Primärdaten an der empirischen Verfügbarkeit. Zur Auswertung wird für jede einzelne Policy zum barrierefreien bzw. -reduzierten Wohnraum geprüft, welche Kategorien zielführend zur Beschreibung der Maßnahme und zur Beurteilung der Varianz zwischen den 16 Ländern sind.

\subsection{Politikfeldanalytische Fallstudien}

Aufbauend auf der Typologie der politischen Maßnahmen werden politikfeldanalytische Fallstudien für die Bundesländer Sachsen-Anhalt und Hessen durchgeführt (vgl. Kapitel 7). Das Ziel ist es, die hinter den Landes-Policies zum barrierefreien bzw. -reduzierten Wohnraum liegenden politischen Prozesse zu verstehen und zu erklären. Mit 